Conclusion The addition of co-trimoxazole therapy to standard treatment for Idiopathic pulmonary fibrosis had no effect on lung function or disease progression but resulted in a fivefold reduction in mortality and was cost-effective at UK thresholds.

\section{Pathophysiology and management of cough S139 COUGH RESPONSES TO TUSSIVE AGENTS IN HEALTH AND DISEASE}

doi:10.1136/thoraxjnl-2011-201054b.139

${ }^{1} \mathrm{~S}$ Khalid, ${ }^{1} \mathrm{R}$ Dockry, ${ }^{1} \mathrm{~K}$ Holt, ${ }^{1} \mathrm{H}$ Sumner, ${ }^{1} \mathrm{D}$ Valdramidou, ${ }^{2} \mathrm{M}$ A Birrell, ${ }^{2} \mathrm{M} \mathrm{G}$ Belvisi, ${ }^{1}$ A Woodcock, ${ }^{1} \mathrm{~J}$ A Smith. ${ }^{1}$ University of Manchester, Manchester, UK; ${ }^{2}$ National Heart and Lung Institute, London, UK

Introduction Capsaicin or citric acid cough challenges have been used as an objective measure of cough reflex sensitivity for many decades. It remains unclear how the response to these agents differs in different diseases and how the response to one cough challenge agent differs from that to another. Prostaglandin E2 is known to result in cough when given as an inhalational agent, but has not been used as inhalational cough challenge agent.

Objectives To assess the ability of individual challenges and combined challenge responses to discriminate between diagnostic groups and healthy volunteers.

Methods We studied 102 subjects, median age 60.0 years (IOR $51.0-65.0$ ) and $50 \%$ female (healthy volunteers $n=21$, healthy smokers $\mathrm{n}=20$, COPD $\mathrm{n}=18$, asthma $\mathrm{n}=22$ and chronic cough $\mathrm{n}=21$ ). A doubling-dose, single inhalation method was used to measure the concentration of capsaicin (CAP), citric acid (CA) and prostaglandin E2 (PGE2) evoking at least 5 coughs (C5) within $15 \mathrm{~s}$ of administration, performed at weekly intervals. The operator was blinded to the challenge agent and each challenge contained 3 randomly interspersed placebo inhalations (saline). Data was analysed by multinomial logistic regression with healthy volunteers used as the reference category.

Results Smokers $(p=0.03)$ and COPD patients $(p=0.003)$ had a significantly higher PGE2 logC5 than healthy volunteers. CA logC5 however was significantly lower in asthma $(p=0.013)$, and chronic cough ( $p=0.001)$ compared with healthy volunteers. CAP logC5 was also significantly lower in chronic cough $(p<0.001)$ but also in COPD ( $p=0.035)$ compared with healthy volunteers. Combining responses to all challenge agents suggested each individual challenge independently predicted the differences between disease groups and healthy volunteers (PGE2 $p<0.001$, CA $p=0.018$ and CAP $p=0.015$ ). Conclusions Cough responses to inhalational cough challenges can discriminate healthy controls from airway diseases. Furthermore, cough challenge agents differ in their ability to distinguish health from disease implying different underlying mechanisms drive coughing in these diagnoses. A combination of cough challenge tests appears to be better at discriminating diagnostic groups compared with any individual test in isolation.

\section{S140 PREDICTORS OF 24-H COUGH FREQUENCY IN ACUTE COUGH}

doi:10.1136/thoraxjnl-2011-201054b.140

${ }^{1} \mathrm{~K}$ K Lee, ${ }^{2} \mathrm{~S}$ Matos, ${ }^{3} \mathrm{D}$ H Evans, ${ }^{4} \mathrm{I}$ Pavord, ${ }^{1} \mathrm{~S} S \mathrm{~S}$ Birring. ${ }^{1}$ Division of Asthma, Allergy and Lung Biology, King's College London, London, UK; ${ }^{2}$ Institute of Electronics and Telematics Engineering (IEETA), University of Aveiro, Aveiro, Portugal; ${ }^{3}$ Department of Medical Physics, Leicester Royal Infirmary, Leicester, UK; ${ }^{4}$ Department of Respiratory Medicine, Institute for Lung Health, Glenfield Hospital, Leicester, UK

Introduction 24-hour cough frequency monitoring is increasingly being used as an outcome measure to evaluate anti-tussive drugs.
The optimal method of identifying patients with a significant cough frequency for inclusion into clinical trials is not known. We investigated a range of cough assessments screening tools that could be used for this purpose.

Methods 35 healthy subjects with acute cough due to upper respiratory infection (median (IOR) age $31(23-35)$ years, 63\% female, median (IOR) duration of cough 4 (2-6) days) were recruited as part of a larger study. All subjects underwent ambulatory 24-h cough frequency $\left(\mathrm{CF}_{24}\right)$ monitoring with the Leicester Cough Monitor, health related quality of life with the Leicester Cough Questionnaire-acute (LCQ) and cough severity visual analogue scale (VAS). Receiver operating characteristic curve analyses were performed for the baseline screening tools LCQ, VAS and cough frequency in first hour $\left(\mathrm{CF}_{1}\right)$ to identify patients with $\mathrm{CF}_{24}=5$, $=7.5$ and $=10$ coughs $/$ hr.

Results The baseline geometric mean (logSD) $\mathrm{CF}_{24}$ was 14.7 (0.5) coughs/hr, $\mathrm{CF}_{1} 25.9$ (0.4) coughs/hr, mean (SEM) VAS 47 (3) mm and LCO 14.3 (0.7). 4, 6 and 11 patients had $\mathrm{CF}_{24}<5,<7.5$ and $<10$ coughs/hr respectively. The area under ROC curve (AUC) for VAS and $\mathrm{LCQ}$ for prediction of $\mathrm{CF}_{24}$ were poor, ranging from 0.51 to 0.58 and 0.50 to 0.68 respectively. The mean (SEM) AUC for $\mathrm{CF}_{1}$ was $0.92(0.05), 0.79(0.10)$ and $0.86(0.06)$ for detecting 24-h cough frequency of $=5,=7.5$ and $=10$ coughs/hr respectively. The sensitivity and specificity for cough frequency recordings over $1 \mathrm{~h}$ to identify patients with significant 24 - $h$ cough frequency are presented in Abstract S140 table 1. A good specificity (to exclude patients with low 24-h cough frequency) was achievable at sensitivities ranging from 69 to $87 \%$.

Abstract S140 Table 1 Accuracy of 1 -h cough frequency $\left(\mathrm{CF}_{1}\right)$ in identifying 24-h cough frequency $\left(\mathrm{CF}_{24}\right)$

\begin{tabular}{llllll}
\hline $\mathbf{C F}_{24}$ detection & $\begin{array}{l}\text { Specificity } \\
\text { level }\end{array}$ & $\begin{array}{l}\text { Sensitivity } \\
(\%)\end{array}$ & $\begin{array}{l}\text { CF }_{1} \text { cut-off } \\
\text { level }(\text { coughs/hr) }\end{array}$ & PPV & NPV \\
\hline$>5$ coughs $/ \mathrm{hr}$ & 100 & 87 & $>12.5$ & 1.00 & 0.50 \\
$>7.5$ coughs $/ \mathrm{hr}$ & 83 & 69 & $>17.5$ & 0.95 & 0.36 \\
$>10$ coughs $/ \mathrm{hr}$ & 92 & 74 & $>20.5$ & 0.94 & 0.65 \\
\hline
\end{tabular}

Conclusion Subjective cough severity and cough-specific quality of life are poor screening tools for predicting objective 24-h cough frequency. However, 1-h cough frequency can be used as a screening tool to identify patients with significant 24 -h cough frequency for inclusion into future clinical trials.

\section{S141 TRPA1 EXPRESSION AND CHARACTERISATION IN PATIENTS WITH CHRONIC COUGH}

doi:10.1136/thoraxjnl-2011-201054b.141

${ }^{1} \mathrm{~S}$ Faruqi, ${ }^{2} \mathrm{~A}$ Campbell, ${ }^{2} \mathrm{~A}$ H Morice. ${ }^{1}$ Russells Hall Hospital, Dudley, UK; ${ }^{2}$ Castle Hill Hospital, Hull, UK

Introduction and Aims The TRPA1 ion channel is thought to have an important role in the cough reflex. It has been demonstrated that agonisation of TRPA1 receptors by inhalation of cinnamaldehyde, a specific agonist of TRPA1, induces cough in normal human volunteers. We wanted to determine the expression and characterisation of the TRPA1 receptor in human lung tissue.

Methods Bronchial biopsies were obtained from patients with the Cough Hypersensitivity Syndrome on fibre-optic bronchoscopy. Biopsies were obtained from a major bronchus. Lung resection samples were obtained from patients undergoing surgery for lung cancer. Dorsal root ganglia were used as a positive control. These tissue samples were analysed by immunohistochemistry with a specific TRPA1 antibody. 